\title{
Références
}

\section{Utiliser les bases bibliographiques bio-cliniques à bon escient (M edline, Embase, etc.)}

Article élaboré collectivement par la rédaction de la revue Prescrire*

\begin{abstract}
Messagesclés
- Lesétudiants, les enseignants et les professionnels de santé peuvent consulter les bases bibliographiques: pour retrouver les références déailléesd'un artide déà connu ; pour réaliser un travail universitai re (mémoire, thèe) ; pour préparer un cours en faculté ou une séance de formation continue; pour élaborer un protocolederechercheou rédiger un articleou un ouvrage. - La base de données américaine M edline est loin d'indexer la totalité des journaux médicaux. Environ $90 \%$ de ces réfé rences bibli ographi ques renvoient à des artides publiés en anglais. U ne minorité de publications présentent des résultats di rectement transposables en dinique, tout en reposant sur un niveau de preuves suffisant. La qualité méthodologique des publications indexées es extrêmement variable. - Les résumés des articles (abstracts) sont en général trop peu informatifs pour juger de la fiabilitédel'étude originale et de sa réelle portée pratique. • Une synthèse dela littérature digne de ce nom nécessite une recherche simultanée dans plusieurs bases, choisies en fonction du sujet considéré. - Chaque basebi bliographique comportedes documentsindexéspar une listede mots désqui lui et propre La syntaxed'interrogation d'une base et les possibilités d'interrogation dépendent du fournisseur d'accès - U ne inter rogation fructueuse requiert l'établi ssement préalable, base par base, d'une listedemots adéquats, le plus souvent en anglais, et d'une sratégie de recherche documentaire consistant en une séri e de combinaisons bien pensées de ces termes entreeux. ${ }^{\circ}$ D ocumenter une synthèse de la littérature ne simprovise pas: il faut soit se former à l'interrogation des bases bibliographiques utilisées, soit recourir aux services d'un documentalise professionnel.
\end{abstract}

M ots clés Recherche documentaire, bibliographie, bases de données; synthèse méthodique.

\section{Key M essages}

- Students, teachers and health care professionals may have to search bibliographical databases in order: to find the full reference of a scientific paper which is already known to them; to complete an academic work, to prepare a lecture in medical school or a session of continuing medical education, to set up a research design or to write an article or a book. - TheAmerican database M edline, freely accessible on the Internet via PubM ed, isfar from indexing all medical journals. Approximately $90 \%$ of its bibliographical content refers to articles published in English. ${ }^{\circ}$ Only a few scientific papers have results that can be directly turned into clinical practice, while being based on a high enough level of evidence. The methodological quality of articlesindexed in $M$ edline is extremely variable. $\bullet$ The summaries of the articles (abstracts) are usually not informative enough to assess the reliability of the original study and its practical relevance. - A rigorous synthesis of the literature requires simultaneous searches in several databases, selected according to the subject. - Each bibliographical database comprises documents indexed according to its own specific list of keywords. Search engines and interfaces vary with the provider. ${ }^{*}$ A fruitful query requires having previ ously set up of a list of adequate keywords, usually in English, and defining a relevant strategy of informati on retrieval. • To build up a valid synthesis of the literature requires specific training or the assistance of a library information scientis.

\section{Keys WordS Information retrieval; bibliography; database; systematic review.}

Pédagogie M édicale $2004 ; 5$ : 52-60

* La version princeps de cet article de synthèse a été publiée originellement dans le supplément du mois de décembre 2003 de la revue Prescrire, consacréà la recherche documentaire dans le contexte des soins primaires et intitulé " Se documenter pour soigner. Choisir les bons outils »'.

Correspondance : D Broclain. La Revue Prescrire, BP 459, 75527 Paris Cedex 11. Téléphone : +331492372 80 mailto:revue@prescrire.org 
Les résultats de l'interrogation d'une base bibliographique se présentent sous forme d'une liste de références. Une référence bibliographique se caractérise par un ensemble de paramètres qui décrivent la publication originale : titre, auteur(s), nom de la revue, année de publication, résumé, mots-clés, type de publication, langue, etc.

\section{Les bases de données bibliographiques ont une structure complexe}

Pour décrire le contenu des documents, chaque base de donnéesfait appel à un vocabulaire contrôlé, c'est-à-dire à uneliste de mots-clés, alias descripteurs, qui lui est propre Lorsque ces listes standardisées et évolutives de descripteurs comportent des relations entre leurstermes (synonymies, termes associés, termes situés hiérarchiquement plus haut (aliastermes génériques) ou plusbas (aliastermes spé cifiques), la listedes mots-clésest alors appelée «thésaurus».

Les champs (titre, résumé, etc.) décrivant le contenu des documents d'unebase bibliographique peuvent être interrogés soit dans le langage librement choisi par l'utilisateur (langage naturel), soit en employant des termes appartenant obligatoirement à la liste de mots-clés propreà la base (langage contrôlé), soit en combinant les deux types de langages, naturel et contrôlé.

La plupart des bases bibliographiques sont désormais accessibles via internet, parfois gratuitement, le plus souvent contre paiement.

Les bases intéressant les professionnels de santése différencient les unes des autres selon : la zone géographique d'édition des publications indexées (Europe, Amérique latine, etc.) ; les domaines de connaissances couverts (soins infirmiers, toxicologie, sida, médecine sportive, histoire de la médecine, etc.) ; les types de documents inclus (articles de presse, publications scientifiques primaires ou secondaires, synthèses méthodiques, guides de pratique, etc.) ; les critères d'inclusion des publications dans la base (existence d'un comité de lecture, niveau de preuves élevé des données publiées, méthode explicite de synthèse de l'information, etc.).

On trouve, sur le site internet de la $\mathrm{D}$ irection des bibliothèques de l'U niversité de M ontréal (http://www.bib. umontreal.ca) ou sur celui del'Institut national de sciences appliquées (IN SA) de Lyon (http://csidoc.insalyon.fr/sapristi/fristi31.html ), des listes répertoriant des bases de données en ligne sur internet, notamment des bases bibliographiques médicales.

\section{L'exemple de Medline}

Il est indispensable de connaître les caractéristiques d'une base bibliographique si I'on veut avoir les meilleures chances de retrouver avec précision ce qu'on y recherche. $\mathrm{N}$ ous prenons $\mathrm{M}$ edline comme exemple, pour illustrer les nombreuses chaussetrapes qui guettent le novice, en gardant à l'esprit que ce qui est vrai pour M edline l'est aussi pour les autres bases bibliographiques.

\section{Plus de 12 millions de références}

M edline (M edical Literature, Analysis, and Retrieval System 0 nline) est la principale base bibliographiquedela National Library of M edicine américaine (N LM ). Elle contient à ce jour plus de 12 millions de références de publications du domaine des sciences de la vie. L'intégration de nouvelles références y est quotidienne: en 2002, environ 2000 références bibliographiques ont été ajoutées à $M$ edline chaque jour ouvrable, avec un apport annuel de 460000 nouvelles citations. C'est la base de données médicales la plus utilisée dans le monde ${ }^{2,3}$.

M edline est la forme informatisée de l'Index M edicus, une série d'ouvrages volumineux dont la naissance remonte à 1879, destinés à indexer les collections de la $\mathrm{N}$ ational Library of M edicine, elle-même fondée en 1836. Les disciplines couvertes par M edline sont variées : sciences fondamentales (biologie moléculaire, biochimie, chimie, etc.), disciplines cliniques (médecine, soins infirmiers, odontologie, médecine vétérinaire, pharmacie, etc.), mais aussi sciences humaines et sociales.

\section{Plusieurs accès}

M edline est interrogeable en ligne depuis 1971. Principal sous-ensemble de PubM ed, base bibliographique plus large, M edlineest accessible via internet depuis 1995, gratuitement et sans inscription préalable, soit à partir de la page d'accueil de la $\mathrm{N}$ ational Library of M edicine (http://www.nlm.nih.gov), soit à partir de PubM ed (http://www.ncbi.nlm.nih.gov/entrez), soit encoreà partir de N LM Gateway (http://gateway.nlm.nih.gov).

M edlineplus, qui apparaît sur la page d'accueil de la $\mathrm{N}$ ational Library of $\mathrm{M}$ edicine, est un portail produit par la $N$ ational Library of M edicine à l'intention du grand public. Cen'est pas une base de données bibliographiques, mais un répertoire de pages $W$ eb sélectionnées pour les patients par la N ational Library of M edicine. 


\section{Références}

PubM ed est une base bibliographique développée par le $\mathrm{N}$ ational Center for Biotechnology I nformation (NCBI) de la $\mathrm{N}$ ational Library of $\mathrm{M}$ edicine, accessible via internet (http://www.ncbi.nlm.nih.gov/entrez), sans autrefrais que ceux de la connexion internet de l'utilisateur. PubM ed permet, entre autres, d'interroger la base de données M edline.

D e plus, PubM ed donne accès à des articles publiés dans des journaux scientifiques de portée générale qui sortent du champ couvert par M edline (par exemple, des articles sur la tectonique des plaques ou sur l'astrophysique), à des articles dont l'indexation dans $M$ edline a été prévue mais n'a pas été encore été mise en œuvre, et à des articles biomédicaux en texte intégral provenant d'une soixantaine de journaux mis en ligne gratuitement dans une base d'archives électronique nommée PubM ed C entral (PM C, http://www.pubmedcentral.nih.gov/). BioM ed Central (BM C) permet un accès gratuit à ses journaux électroniques sur son propre site internet (http://www.biomedcentral.com) et les archive simultanément dans PubM ed Central. BioM ed Central est une maison d'édition qui défend le principe du libre accès aux articles de recherche, convaincu que les communications scientifiques doivent être largement et facilement accessibles. Pour cette raison, BioM ed Central propose des frais de publication plutôt qu'un système d'abonnement. Autrement dit, ce sont les auteurs qui payent.

$D$ ansla plupart des bibliothèques médicales, M edlinepeut également être interrogée, à travers différentes interfaces, via des prestatai res spécialisés dans les bases de données (par exemple OVID, SilverPlatter, etc.) ou de nombreux organismes publics ou privés.

\section{Les limites inhérentes à Medline}

L'indexation de publications dans M edline a démarré en 1966.

\section{Essentiellement des périodiques}

Les livres de médecine ne sont pas indexés dans M edline. A l'exception de la période allant de 1976 à 1981, en tant quetypes de documents, les comptes rendus de congrès et les communications aux sociétés savantes ne sont pas systématiquement intégrés dans M edline. Ils le sont toutefois si ces textes ont été publiés dans des journaux indexés dans M edline.

\section{Résumés inconstants}

La plupart des revues indexées dans M edline le sont à la suite d'une décision consultative du Literature Sel ection Technical Review Committee (LSTRC), un panel d'experts extérieurs à la $\mathrm{N}$ ational Library of $\mathrm{M}$ edicine, affiliés au $\mathrm{N}$ ational Institutes of $\mathrm{H}$ ealth américain $(\mathrm{N} \mathrm{IH})^{4}$. D ans certains domaines spécialisés, la N ati onal Library of M edicine délègue à des organismes partenai res (par exemple I'American D ental Association, I'American Hospital A ssociation ou I'E mergency Care Research I nstitute (ECRI) le choix des publications intégrables dans M edline (" spe cial collaborative arrangements »). Toutes années confondues, la moitié environ des références que comporte M edlinesont accompagnées d'un résumé $(53 \%)$. D ansles années 2000, 78 \% des références comportent un résumé. Plus de $75 \%$ des résumés sont ceux de la publication originale et ont été rédigés par ses auteurs 5 .

\section{Américanocentrisme}

Les articles référencés dans M edline proviennent de plus de 4600 journaux de 70 pays, écrits dans une trentainede langues distinctes. Sur la période allant de 1997 à 2001, près de $89 \%$ des références indexées dans M edline ont été publiées en anglais, plus de la moitié provenant des ÉtatsU nis d'A mériqué. La part des articles de langue anglaise ne cesse de croître (91\% des articles référencés publiés en 2002-2003 sont en anglais).

$M$ edline est loin d'indexer la totalité des publications médicales: des bases de données bibliographiques telles qu'E mbase (http://www.embase.com) ou Pascal (http://www.inist.fr) indexent denombreux journaux éditésen Europequi ne sont pasindexés dans $M$ edlineet réfé rencent volontiers les articles rédigés dans des langues européennes autres que l'anglais $s^{6,7}$.

\section{Une indexation complexe}

Le vocabulaire contrôlé utilisé par le personnel de la $\mathrm{N}$ ational Library of $\mathrm{M}$ edicinepour indexer le contenu des documents référencés dans M edline (http://www.nlm.nih. gov/mesh) est appelé le M edical Subject $H$ eadings, alias $\mathrm{M}$ eSH . Le M eSH est du domaine public : il est également employé comme vocabulai re d'indexation par de nombreuses bases de données médicales, bibliographiques ou textuelles, dans le monde (Cliniweb, The Cochrane Library, D ocC ismef, DARE, etc.).

Le M eSH est révisé chaque année : des termes périmés sont retirés, de nouveaux termes sont ajoutés. Chaque terme du M eSH comporte une notice descriptive qui précise la date de son introduction dans le thésaurus, le terme utilisé avant son introduction pour indexer un 
articlesur le même sujet, le sens exact qui doit lui êtreatta ché, les confusions possibles à éviter, les renvois possibles vers d'autres termes $M$ eSH (termes génériques, termes spécifiques, synonymies) $)^{8}$. Pour utiliser à bon escient un terme du M eSH au moment d'interroger M edline ou PubM ed, il est nécessaire de connaître non seulement la définition qui en est donnée, mais également la définition des termes situés plus haut (termes génériques) et plus bas (termes spécifiques) dans l'arborescence du thésaurus.

\section{Hiérarchie}

Certaines interfaces d'interrogation de $M$ edline, telles PubM ed, pratiquent I' « explosion » automatique des descripteurs utilisés pour interroger la base de données: cel a signifie que le système utilisera non seulement les descripteurs choisis par l'utilisateur, mais égal ement tous les termes spécifiques associés à ces descripteurs, situés plus bas dans la hiérarchie du M eSH . Si l'interfacene pratique pas I' " explosion », seuls les descripteurs choisis par I'utilisateur seront mobilisés pour rechercher des références bibliographiques, en omettant de chercher les articles abordant des aspects plus spécifiques de la question.

\section{Qualificatifs \\ Les termes M eSH peuvent être associésà une série de qua- lificatifs (sub-headings) qui en décrivent un aspect particu- lier : effets indésirables, diagnostic, chirurgie, ethnologie, etc. \\ En 2003, le M eSH totalise 83 qualificatifs distincts, chaqueterme du M eSH étant assorti d'une liste des quali- ficatifs qui peuvent lui êtrelogiquement associés. L'emploi des qualificatifs dans les stratégies de recherche restreint le nombre de références affichées dans les résultats de l'inter- rogation de M edline. \\ Concevoir une stratégie d'inter rogation performante demande du savoir-faire}

La recherche de références bibliographiques dans $M$ edline peut se faire notamment par nom d'auteur, par mot du titre ou du résumé, par nom de journal, par mots-clés du $\mathrm{M}$ eSH , avec ou sans qualificatifs, ou par une combinaison deplusieurs entrées d'indexation.

G râce à son langage d'interrogation contrôlé, le M eSH permet d'optimiser la recherche documentaire dans M edline et de retrouver la majorité des références sur un sujet donné, qui sont présentes dans M edline.

II ne faut pas perdre de vue que l'indexation réalisée par le personnel de la $\mathrm{N}$ ational Library of M edicine comporte toujours des erreurs et quel'usage exclusif des descripteurs dans une stratégie d'interrogation ne garantit pas de retrouver la totalité des références. L'approche optimale combine langage naturel et langage contrôlés.

\section{Utilisateurs expérimentés versus utilisateurs novices}

U ne étude a comparé les résultats d'une série d'interrogations en ligne de M edline, selon qu'elles avaient été mises en œuvre par des utilisateurs expérimentés ou bien par des utilisateurs novices ${ }^{10}$. Les références bibliographiques recherchées dans $M$ edline concernaient des questions identiques pour les deux groupes.

Les novices n'ont retrouvé que la moitié $(55 \%)$ des réfé rences pertinentes retrouvées par les documentalistes professionnels; de plus, toujours en comparaison avec les utilisateurs expérimentés, les novices ont obtenu un surcroît de $50 \%$ de références non pertinentes.

Les utilisateurs novices, qui ne connaissaient pas les résultats de la comparai son, se sont déclarés sati faits des résultats de leur recherche documentaire ${ }^{10}$.

\section{Formation efficace}

Les auteurs ont ensuiteentrepris de former ces utilisateurs novices à l'interrogation de $M$ edline et ont montré, dans une seconde étude, qu'une formation de deux heures suivie d'un minimum d'entraînement (la pratique d'au moins quatre recherches documentaires dans $\mathrm{M}$ edl ine, à intervalles rapprochés) permettait à ces derniers d'atteindre un niveau deperformances proche de celui desutilisateurs expérimentés ${ }^{11}$.

\section{Références faussement absentes}

$D$ 'autres études se sont attachées à anal yser comment avaient étéformulées les stratégies d'interrogation débouchant sur un messageannonçant qu'aucune référencen'aété trouvée. Ces « réponses vides » sont exceptionnellement la conséquence de l'absence totale de référence bibliographique sur le sujet intéressant l'utilisateur. M edline renvoie en général ce type de message pour des raisons diverses: l'emploi abusif de l'opérateur booléen «AN D »dans le libelléde la question adressée à M edline; un nom d'auteur ou un mot-clé mal orthographié; une ponctuation ou une troncature inappropriées.

D 'autres types d'erreurs classiques sont l'emploi d'un descripteur M eSH inapproprié, , $^{3}$. Exemples: l'utilisation erronée du terme «pediatrics» qui ne désignepas lesmaladies de l'enfant, maislemétier de pédiatreet la pédiatrieen tant que discipline spécialisée; ; l'emploi du terme paracetamol, qui a pourtant statut de dénomination commune internationale 


\section{Références}

(DCI), au lieu du terme M eSH acetaminophen ; le fait de ne pas lier par des «OR » destermes associés ou des termes équivalents; et l'utilisation de mots rarement employés en anglais (exemple: hemoccult est en fait un nom de marque, (H emoccult ${ }^{\circledR}$ ) et, en anglais, l'expression communément utilisée est « fecal occult blood tes » alias FO BT.

\section{Attention aux biais de recherche documentaire}

Une synthèse impartial e des travaux de recherche effectués sur un sujet donné consiste à faire le point, sans parti pris, sur une question donnée, en examinant l'ensemble des données produites jusquelà dans le monde.

Un rapport britannique a examiné en détail l'impact de trois types de biais mettant en péril la validité des conclusions d'une synthèse de connai ssances ${ }^{12}$. Ces biais ont en commun de ne prendre en compte qu'une partie seulement de la masse des recherches effectuées sur le sujet en question, la synthèse de connaissances excluant de ce fait un certain nombre de données, pourtant bien dans le sujet. Ces biais sont désignés, en anglais, sous le nom de reporting biases.

\section{Le biais de publication (« publication bias»)}

D es auteurs, des financeurs et des éditeurs, peuvent décider de publier ou non une étude selon la nature de ses résultats. L'absence de différence statistiquement significative dans les résultats d'une étude (on parle d'étude négative) conduit fréquemment à ne pas la publier ${ }^{13}$.

\section{Le biais linguistique}

La langue choisie par les auteurs pour publier leur travail dépend souvent des conclusions de leur étude. Pour les auteurs des pays non anglophones, la tendance actuelle est de publier à l'étranger et en anglais s'ils obtiennent des résultats « positifs » (mise en évidence d'une différence significative), et de publier dans leur pays et dans leur langue maternelle si leurs résultats sont « négatifs ». De nombreuses méta-analyses restreignant les études incluses à la langue anglaise, le biais linguistique peut en fausser les conclusions.

Une équipe québécoise a ainsi examiné toutes les métaanalyses publiées par huit revues scientifiques majeures sur une période donnée, et refait, le cas échéant, les recherches bibliographiques mentionnées dans les méta-analyses en incluant, cette fois, les articles publiés dans des langues autres que l'anglais. Sur les 36 méta-analyses analysées,
$28(73 \%)$ avaient restreint leur stratégie de recherche bibliographique à des articles en anglais. Parmi les nouveaux articles retrouvés par les recherches documentaires sans restriction linguistique des auteurs québécois, 11 ont été identifiés comme susceptibles de modifier les conclusions de 7 des méta-analyses ${ }^{14}$.

\section{Lebiais Medline}

Un autre biais consiste à ne pas prendre en compte les données non indexées dans M edline, qu'elles soient en anglais ou pas. D e nombreuses revues, non prises en compte dans M edline, le sont dans d'autres bases de données bibliographiques (notamment Embase, Pascal, etc.). L'I nternational Journal of 0 bstetric Anesthesia, par exemple, n'est pas indexé dans M edline, alors qu'il a publié plus de 80 essais randomisés ${ }^{15}$. Par ailleurs, de nombreuses revues médicales publiant des études cliniques ou épidémiologiques de qualité, notamment des revues éditées dans des pays commel'Inde ou la Chine, ne sont pas indexées dans les bases bibliographiques.

\section{Lebiais «FUTON »}

L'accès en ligne, gratuit ou payant, aux bases de données viainternet, et la possibilitédetéléchargement d'articles en texteintégral facilitent indéniablement l'accès à l'information médicale. $M$ ais ces avancées technologiques portent avec elles le risque de nouveaux biais dans les synthèses de connaissances médicales.

Il est ainsi tentant de se limiter à rechercher des publications accessibles sur internet en texte intégral, et, si possible, gratuites. Lebiais «FUTON » (Full Text On the N et bias) affecte les conclusions des synthèses de la littérature tout autant que le biais de publication, le biais linguistique ou le biais M edline ${ }^{15}$.

Une équipe de documentalistes a analysé pendant deux semaines les demandes d'articles provenant des chefs de service d'un hôpital nord-américain : en décembre 2002, sur les 323 articles demandés, près de la moitié $(47 \%)$ n'étaient pas accessibles en ligne via internet, et $7 \%$ seulement des articles accessibles en ligne pouvaient être télé chargés gratuitement ${ }^{16}$.

\section{Le biais «N 0 abstract available»}

Un autre biais consiste à ne pas commander (et donc à ne pas lire) les articles lorsque leur référence n'est pas accompagnée d'un résumé (abstract) dans les bases bibliographiques. Le No abstract Available bias affecte notamment les articles en cours d'indexation, les éditoriaux et les 


\section{Conseils d'utilisation des bases de données bibliographiques}

\section{Se poser des questions indispensables avant toute recherche}

Un petit nombre de questions permettent de préciser le projet de recherche dans les bases bibliographiques. Q uel est l'objectif ? R etrouver certaines références ou réaliser une synthèse méthodique de la littérature sur un sujet donné?

D e quels types de documents a-t-on besoin ? D 'articles publiés ? D 'abstracts de congrès ? D e rapports non publiés? D e comptes rendus d'essais ou de synthèses méthodiques?

$D$ ans quel domaine de connai ssances va-t-on rechercher I'information ? Les soins dentaires ? La toxicologie animale? Les aides techniques? La thérapeutique humaine? etc.

Cherchet-on des informations récentes, anciennes ou les deux?

D e quels moyens (temps et argent) disposet-on ? A-t-on les moyens financiers de payer l'accès aux bases de données bibliographiques requises et d'acheter les articles potentiellement utiles ? A-t-on le temps de se déplacer à la bibliothèque la plus proche pour faire des photocopies? etc.

\section{C onsulter des guides d'utilisation et modes d'emploi - D es outils peuvent aider la recherche}

Guides de la N ational Library of M edicine(N LM ). La $\mathrm{N}$ ational Library of M edicine met en ligne un mode d'emploi sur les modalités d'interrogation dePubmed, et donc de M edline (http://www.ncbi.nlm.nih.gov/entrez/ query/static/help/pmhelp.html).

La National Library of M edicine propose également un manuel de travaux pratiques (téléchargeable au format PD F ou Word), qui contient des exercices commentés et corrigés (http://www.nlm.nih.gov/pubs/web_based. html).

\section{- Portail des modes d'emploi}

La H ealth Sciences Library, bibliothèque médicale universitaire de Buffalo aux États-Unis d'Amérique, met en ligne un portail des modes d'emploi en anglais de Medline, PubM ed, et autres bases de données (http://ublib.buffalo.edu/libraries/units/hs//ref/tutorials.html.

\section{- Cours en français}

L'U nité régionale de formation à l'information scientifique et technique de Paris (U RFIST Paris, É cole nationale des chartes) propose un cours bien fait, en français, complété d'un test de connaissances avec autocorrection instantanée, sur l'interrogation de M edline via PubM ed

(http://www.ccr.jussieu.fr/urfist/biolo/bioguide2/medline/medline.htm).

La bibliothèque de la faculté de médecine de Bicêtre (France) propose une page web en français, dédiée à I'apprentissage de l'interrogation de M edline via PubM ed (http://www.upsud.fr/Biblikb.nsf/formations.htm ! 0 penPagepubmed).

La direction des bibliothèques de l'université de $M$ ontréal ( $Q$ uébec) a conçu une page $W$ eb en français d'initiation à l'utilisation de PubM ed (http://www.bib. umontreal.ca/SA/caps35.htm).

\section{Saisir - rechercher des synthèses méthodiques et des publications primaires à haut niveau de preuves dans PubMed}

La section «Clinical queries» de PubM ed donne accès à une interface d'interrogation particulièrequi permet : - soit de restreindre sa recherche aux synthèses méthodiques présentes dans PubM ed

(en anglais, «systematic reviews ») ;

- soit de ne chercher que des articles ayant à la fois un intérêt clinique et un niveau de preuves assez élevés. L'utilisateur peut activer des filtres méthodologiques, au choix, dans les domaines thérapeutique, diagnostique, pronostique ou étiologique. Un choix supplé mentaire est proposé entre une recherche documentaire sensible, dont les résultats contiendront la totalité les articles pertinents, au prix d'un surcroît de bruit documentaire, soit une recherche documentaire spécifique, présentant peu de bruit documentaire, mais au risque de passer à côté d'un certain nombre d'articles pertinents. La sensibilité des filtres méthodologiques de PubM ed dépasse $80 \%$, et leur spécificité avoisine ou dépasse $97 \%{ }^{1}$. 


\section{Références}

\section{Interroger PubMed en français}

Voici trois sites qui permettent d'interroger $M$ edline via

Pubmed, en français.

\section{- Le MeSH en français de l'Inserm}

Suite à un accord avec la National Library of M edicine, I'Inserm traduit en français et met à jour le vocabulaire du M eSH . Cette traduction française est disponible sur le site du D épartement de l'information scientifique et de la communication (DISC) de l'Inserm. Ce site permet de retrouver les termes $\mathrm{M}$ eSH anglais que l'on doit utiliser pour interroger M edline. II est possible d'y rechercher un terme pour voir les informations (définition, etc.) et les relations (synonymes, termes associés) le concernant, le situer dans son environnement hiérarchiqueet rechercher directement les références bibliographiques correspondantes sur la base PubM ed. O n trouve aussi sur le site du DISC les modifications annuelles du M eSH (termes nouveaux, modifiés ou supprimés).

\section{- L'interface $\mathrm{H} O \mathrm{~N}$ sélect}

HON sélect (http://www.hon.ch/H O N select/ index_f.html) est une interface d'interrogation de PubM ed mise au point par la Fondation $\mathrm{H}$ ealth $\mathrm{O} n$ the
Net. C ette interface en français permet d'interroger la base PubM ed à l'aide du M eSH en version française et donne la possibilité de restreindre ses recherches à des articles debonnequalité méthodologique grâce aux « dinical queries». M alheureusement, l'option permettant de retrouver les synthèses méthodiques dans PubM ed (synthèses Cochrane, etc.) n'est pas disponible.

\section{- L 'interface CliniWeb International}

CliniWeb International (http://www.ohsu.edu/cliniweb/ search.html) est uneinterface d'interrogation deM edline via PubM ed. L'interrogation en français est possible La restriction aux filtres méthodologiques de PubM ed (dinical queries), incluant la recherche de synthèses méthodiques, est permise. L'interrogation se lance à partir d'un écran qui affichel'arborescencedu M eSH , ce qui permet d'interroger aisément des termes plus spécifiques ou des termes plus larges dans la hiérarchie du M eSH .

1- Haynes RB, Wilczynski N , M cKibbon KA, Walker CJ, Sinclair JC. D evel oping optimal search strategi es for detecting dinically sound studiesin M edline. J Am M ed Inform Assoc $1994 ; 1$ : 447-58. lettres à la Rédaction, dont l'intérêt potentiel n'est pas moindre du seul fait que ces articles ne comportent pas de résumé ${ }^{15}$. O n peut y trouver par exemple des réflexionsou des critiques méthodologiques décisives.

\section{Conclusion : la qualité ne simprovise pas}

Q uece soit pour un travail universitaire ou pour un travail de recherche, une synthèse de la littérature correctement conçue ne s'improvise pas : pour éviter d'avoir une vue parcellaire et biai sée sur le sujet étudié, il ne faut pas hésiter à se former à l'interrogation correcte des bases bibliographiques utilisées, ou bien recourir aux services d'un(e) documentaliste.

Pour le professionnel de santé ou l'étudiant, la consultation des modes d'emploi version papier ou des aides en ligne des interfaces d'interrogation des bases bibliographiques est un préalable obligatoire à leur interrogation car c'est le seul moyen permettant de savoir comment libeller ses questions de la bonne manière et d'obtenir des réponses pertinentes et aussi complètes que possible.

Tous comptes faits, pour répondre à une question qui se pose à propos des soins, mieux vaut se concentrer sur la recherche de documents synthétiques élaborés selon une méthodologie qui en garantit la qualité

Le sommaire du supplément de la revue Prescrire («Se documenter pour soigner. Choisir les bons outils»), dans lequel a été publié la version princeps de cet article, peut être consultéà l'adresse suivante: http://www. prescrire.org/sommaire/N 245.php\#supplt

Pour commander le fascicule: http://www.prescrire. org/signature/productions/supplements.php 


\section{Les bases de données bibliographiques en éducation médicale}

$N$ ote d'information complémentaireélaborée par la rédaction de Pédagogie M édicale

Les problèmesposés par la consultation des bases de données bibliographiques en éducation médicale sont les mêmes que ceux rencontrés dans le champ des références bio-cliniques; les précautions à prendre et les pièges à éviter sont similaires.

En revanche les bases de données concernées sont diffé rentes.

La base de données Medline référence les articles qui sont publiés dans plusieurs revues spécial isées en éducation médicale, telles que Academic M edicine, Advances in $\mathrm{H}$ ealth Sciences Education, M edical Education, M edical Teacher, Teaching and Learning in M edicine.

Cette base donne égal ement accès aux articles d'éducation médical e qui sont publiés périodiquement dans les grandes revues médicales bio-cliniques généralistes, telles que le British M edical Journal, The Lancet, les Annals of Internal M edicine, leJAM A.

D 'autres bases de données sont spécifiques du champ des sciences de l'éducation. Elles constituent des ressources tout à fait pertinentes pour toutes les personnes concernées par la pédagogie universitaire, l'enseignement supérieur et la formation professionnelle. $0 \mathrm{n}$ peut notamment citer parmi elles:

- Le Educational Resources Information Center (ERIC), développé par le Département d'Education des EtatsU nis, qui est la plus importante base de données mondiale dans le domaine de l'éducation. Elle compile les références de plus d'un million de résumés depuis 1966. CommeM edline, elle est accessible via plusieurs interfaces, comme par exemple The Educator's Reference D esk (http://www.eduref.org/Eric/) ou SearchERIC. org (http://searcheric.org/)
- Le British Education index (http://www.leeds.ac.uk/ bei/), portail qui donne accès à plusieurs bases de données spécial isées dont le British Education Internet Resource Catalogue et Education 0 nline, qui héberge notamment les ressources en ligne de The European Conference on Educational Research, The Scottish Educational Research Association et The British Educational Research Association.

- The European Education Thesaurus (EET) qui est développé conjointement par la Commission Européenne et par le Conseil de l'Europe. II est accessible via son portail internet Eurydice (http://www.eurydice.org/ accueil_menu/en/frame set_menu.html). Eurydice met à la disposition de ses usagers une base de données unique et très détaillée sur tous les systèmes éducatifs couverts par le réseau : Eurybase.

La Collaboration BEME (Best Evidence M edical Education) (http://www.bemecollaboration.org/), initiée depuis 1999, tentetransposer au domaine del'éducation médicale la démarche développée par la collaboration Cochrane dans le domaine bio-clinique et thérapeutique. A l'instar de la démarche promue par le courant de I'Evidence-based medicine, elle entend favoriser des pra tiques éducatives en formation médicale qui soient davantage fondées sur des faits démontrés plutôt que sur des arguments d'opinion ou d'autorité.

Parmi les outils dont ellese doteà cette fin, la production de revues systématiques faisant l'état des lieux scientifique des grandes questions d'actualité en éducation est en cours de développement.

Un dossier thématique à paraître dans Pédagogie médicale, concernant les aspects conceptuels et opérationnels de cette démarche, est en projet ; il devrait être disponible dans quelques mois. 


\section{Utiliser les bases bibliographiques bio-diniques..}

\section{Références}

1. Prescrire Rédaction. U tiliser les bases bibliographiques à bon escient (M edline, Embase, etc.). Rev Prescrire 2003; 23 (245 Suppl.) : 891-895.

2. Hersh WR Information retrieval : a health and biomedical perspective 2nd ed. N ew York : Springer 2003.

3. Hersh WR, H ickam DH . How well do physicians use electronic information retrieval systems? A framework for investigation and systematic review. JAM A $1998 ; 280$ : 1347-1352.

4. National Library of M edicine Fact sheet. Journal Selection for Index M edicus/M edline, 2003. D isponible sur : http://www.nlm.nih.gov/pubs/factsheets/jsel.html.

5. NLM Bibliographic services division. M edline: N umber of citations to English language articles; Number of citations containing abstracts, 2003. Disponible sur : http://www.nlm.nih.gov/bsd/medline_lang_distr.html.

6. Elsevier Science. About Embase.com, 2003. Disponible sur : http://www.embase.com/about

7. Fagherazzi-Pagel H, FohannoV, M orel-Pair C. L'INIST/CN RS, du document scientifiqueà la veille, un accès privilégié à l'information biomédicale internationale. Revue M édicale de l'A ssurance M aladie 2001 ; 32 : 167-175.

8. D ailland F. Aide mémoire d'indexation. M eSH (M edical Subject $H$ eadings) et FM eSH (version française) pour lecatalogage Paris: IN SERM -DISC (D épartement de l'information scientifique et de la communication), H ôpital de Bicêtre, Le Kremlin Bicêtre, 2002.
9. G reenhalgh T. Savoir lire un articlemédical pour décider. La médecine fondée sur les niveaux de preuve au quotidien, Meudon : RanD, 2000.

10. Haynes RB, M K Kibbon KA, Walker CJ, Ryan N, Fitzgerald $D$, Ramsden M F. Online access to $M$ edline in clinical settings. A study of use and usefulness. Ann Intern M ed 1990; $112: 78-84$.

11. Haynes RB, Johnston M E, M CKibbon KA, Walker CJ, Willan AR. A program to enhance clinical use of $M$ edline. A randomized controlled trial. J Curr Clin Trials 1993; D oc No 56 [Online]

12. Egger $M$, Juni $P, B$ artlett $C J$, $H$ olenstein $F$, Sterne JAC. H ow important are comprehensive literature searches and the assessment of trial quality in systematic reviews? Empirical study H ealth Technol Assess $2003 ; 7$ : 1-32. D isponible sur : http://www.ncchta.org/fullmonol mon701.pdf

13. ChapuisF. Les biais de publication Rev Prescrire 1994 ; $14: 718-720$.

14. Grégoire G, Derderian F, Le Lorier J. Selecting thelanguage of the publications included in a meta-analysis: is there a Tower of Babel bias?] Clin Epidemiol 1995 ; 48 : 159-163.

15. Went R. Visibility of research : FU TON bias Lance 2002 ; $360: 1256$.

16. Internet spawns new potential biases in assessing evidence. $H$ ealth Technol Assessment N ews (ECRI) 2003 ; 4: 1-2. 